ISSN 1112-9867

http://www.jfas.info

\title{
A MULTI-DEVELOPING-COUNTRY COMPARISON OF THE LEE-CARTER MODEL FOR MORTALITY AND LIFE EXPECTANCY PROJECTIONS
}

\author{
S. Shair ${ }^{*}$, F. Akmal and N. Nordin \\ Centre for Actuarial Studies, Faculty of Computer and Mathematical Sciences, \\ UniversitiTeknologi MARA, 40400 Shah Alam, Selangor, Malaysia
}

Published online: 10 November 2017

\begin{abstract}
This study evaluate the Lee-Carter model for projecting age and gender specific mortality rates and life expectancy at births in four developing countries namely Malaysia, Indonesia, Thailand and Singapore. Although the Lee-Carter model has been successfully applied in many developed countries, little information is obtained on the suitability of the model for developing countries. We fit age and gender specific mortality rates of each developing country from 1960 to 1989 into the model and forecast over the evaluation period from 1990 to 2015. The forecast values are then compared with actual rates over the same period to estimate the out-sample forecast errors. The mean forecast errors of log mortality rates show that the Lee-Carter model works better in Malaysia, Indonesia and Thailand, than in Singapore. In terms of life expectancy at births, the Lee-Carter model consistently more accurate for females than that of males.
\end{abstract}

Keywords: mortality; life expectancy; Lee-Carter model; population ageing.

Author Correspondence, e-mail: syazreen@tmsk.uitm.edu.my

doi: http://dx.doi.org/10.4314/jfas.v9i6s.29 


\section{INTRODUCTION}

An increase in human life expectancy together with a decrease in fertility rates over the last decades has resulted in a social phenomenon known as population ageing. This demographic issue becomes a central concern in most developed countries and in a growing number developing nations. As a result, accurate life expectancy and mortality estimations are increasingly important for planning purposes in particular to ensuring social services and financial assistance can support a growing number of people. To meet this challenge, mortality forecasting model is the key tool. Despite its name, the model is not primarily used to forecast deaths but it is also useful to forecast life expectancy of population.

Mortality forecasting models have been developed significantly over the last decades. For comprehensive reviews on the development of mortality forecasting models [1]. Previously, mortality models were mostly relied on subjective opinion of experts about future trends [2]. However, forecasters' bias judgments have been reduced with constant improvement of extrapolative models-statistical models that make use of past data and assume a common historical pattern will continue in the future.The Lee-Carter model [3] is an extrapolative model that accounts for a decline in mortality pattern when forecasting mortality rates. For the U.S data, the Lee-Carter model is successful in term of accuracy as the forecasts are reported more accurate than the U.S Social Security Administration [4]. Since then, the Lee-Carter model received extensive recognition and has been applied in many developed countries.

However, despite the Lee-Carter model's success in the US and other developed countries, a study from [5] shows that the Lee-Carter's model assumptions were invalid in some countries such as Australia. The application of the Lee-Carter model in developing countries is limited, thuslittle information is known how accurate the model is when using developing countries' data. Hence, it is of interest to this research to report the performance of the Lee-Carter model using developing countries' mortality data.

This research aims to evaluate the accuracy of the Lee-Carter model to forecast age and gender specific mortality rates and gender specific life expectancy at births in four developing countries such as Malaysia, Indonesia, Thailand and Singapore. It is noteworthy that the selection of these countries are due to they are located in the same Southeast Asia region and been grouped under the same developing economies category. The out-sample forecast errors 
of mortality rates and life expectancy at births will be estimated and compared between methods and countries.

\section{DATA AND METHODS}

\subsection{Data}

We collected central age and gender specific mortality rates for Malaysian population from the Department of Statistics Malaysia (DoSM). The mortality data of Malaysian population are available from year 1957 to 2015 and from age 0 to 85 years old. Similar type of data for Indonesia, Thailand and Singapore were obtained from the World Population Prospects, 2015 Revision [6] which available from 1950 to 2015. For consistency, we apply the same observation period for each country that is from 1960 to 2015.

The dynamics of age and gender specific mortality rates in four developing countries are presented in Fig. 1 for males and Fig. 2 for females. Both figures clearly depict an increasing mortality rates over the age groups with the highest mortality occurs in the oldest old age group. As results of medical and health care improvement in developing countries, there has been a continuing decline in mortality rates from red to purple curves (from 1960 to 2015). 
Malaysia males

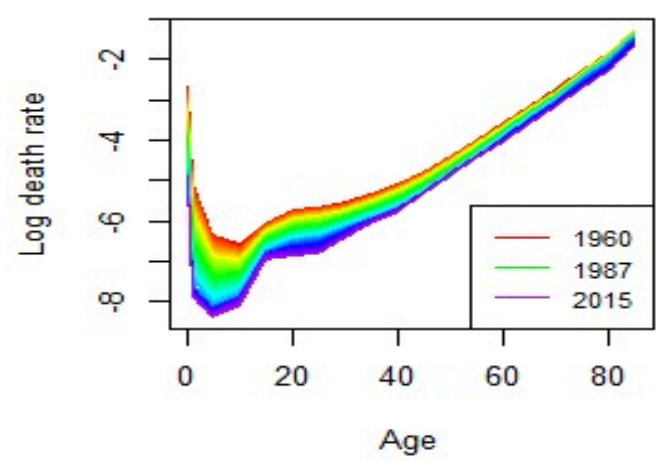

Thailand males

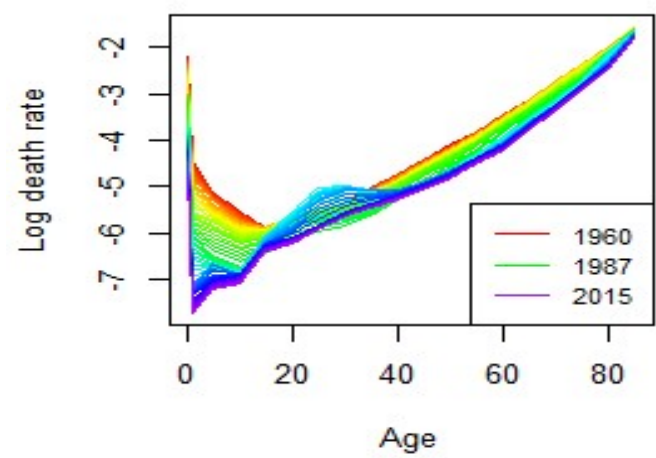

Indonesia males

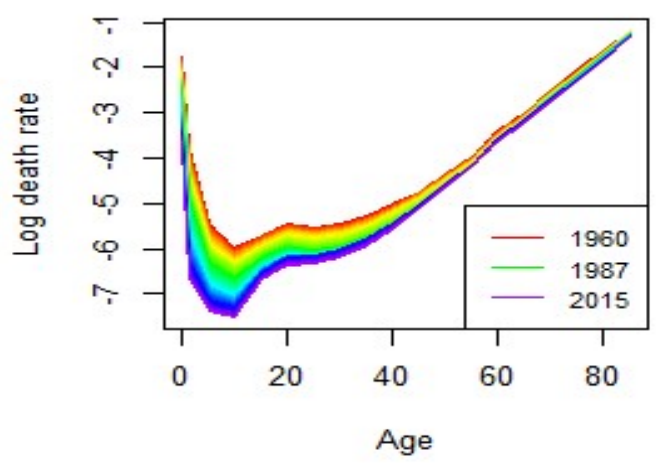

Singapore males

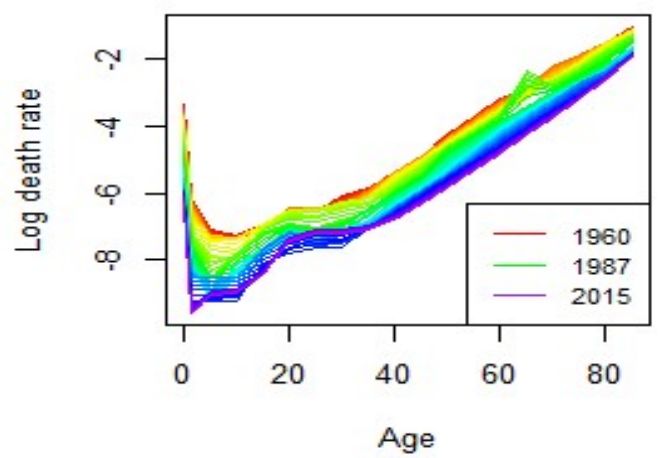

Fig.1. The rainbow plots of log age-specific mortality rates for males in Malaysia and Indonesia (above) and Thailand and Singapore (below) from 1960 to 2015

Fig. 1 and Fig. 2 show that the rate of mortality decline is varied by gender and country. For example, the mortality of Indonesian males aged between 45 and 85 years old has been decreasing at a slower rate than that of males of the same group from other countries. This result is showed by a smaller gap between red and purple curves for Indonesian males (top-left panel of Fig. 1). On the other hand, the mortality of Malaysian females aged 15 to 30 has been decreasing at higher rates compared to other countries, leading to a wider gap between red and purple curves (see top-right panel of Fig. 2). There is inconsistency in mortality patterns between males and females in which the accident humps can only be seen among young to adult males between 15 and 39 years old, and almost nonexistent for females in the same group. This result is in line with the fact that males out-numbered females for motor accident fatalities [7]. 
Malaysia females

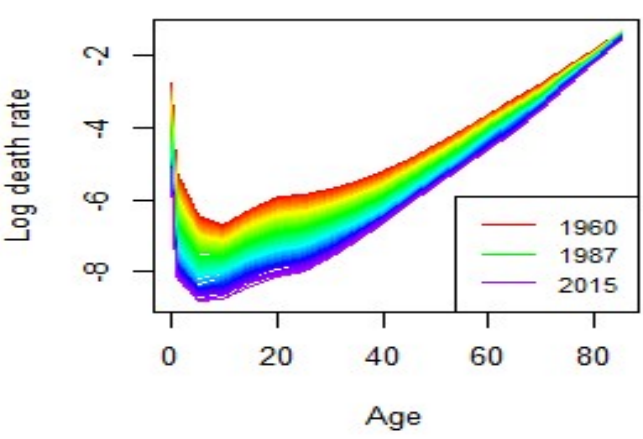

Thailand females

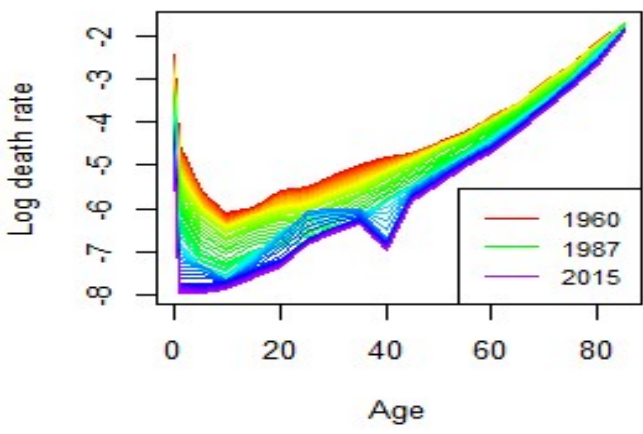

Indonesia females

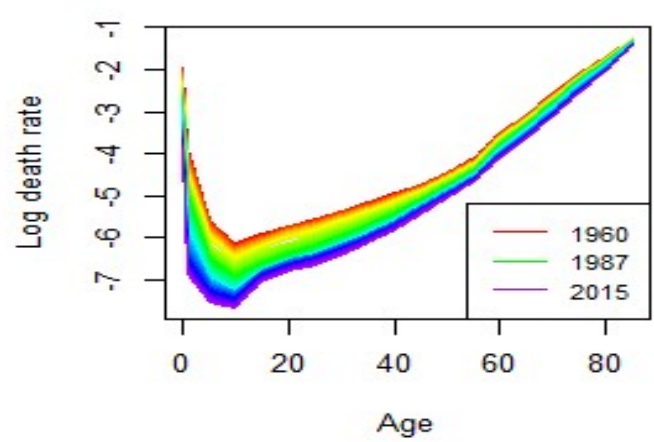

Singapore females

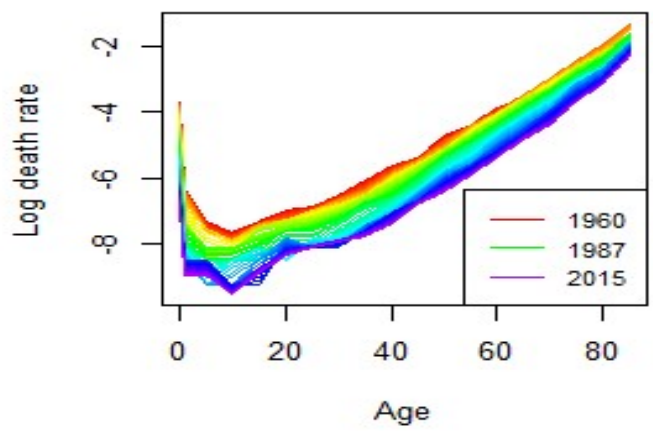

Fig.2. The rainbow plots of log age-specific mortality rates for females in Malaysia and Indonesia (above) and Thailand and Singapore (below) from 1960 to 2015

\subsection{The Lee-Carter Model}

The Lee-Carter model is a well-known method for mortality forecasting due to its ability to produce accurate results yet it is simple to apply. The Lee-Carter model is described as follows:

$$
\ln \left(m_{x, t}\right)=a_{x}+b_{x} k_{t}+\varepsilon_{x, t}
$$

where $m_{x, t}$ is the central death rate for age $x$ in year $t$. The $a_{x}$ variable is the average of $\ln \left(m_{x, t}\right)$ over the years and $b_{x}$ and $k_{t}$ are known as the age-component and mortality index respectively. These $b_{x}$ and $k_{t}$ variables are estimated using a singular value decomposition of $\left[\ln \left(m_{x, t}\right)-a_{x}\right]$ matrix, subject to two constraints which are $\sum_{x} b_{x}=1$ and $\sum_{t} k_{t}=0$.

The Lee-Carter model assumes that the $k_{t}$ variable will display a linear decreasing pattern 
over time, hence a simple random walk with drift is used to forecast $k_{t}$. The random walk with drift model is defined as below:

$$
k_{t}=k_{t-1}+d+\xi_{t}
$$

where $d$ is the average annual change in $k_{t}$ and $\xi_{t}$ are the respective errors.

The forecast of $\log$ of age and gender specific mortality rates, $\ln \left(m_{x, t}\right)$ are obtained from Equation (1) using the forecast values of $k_{t}$ and the estimated values of $a_{x}$ and $b_{x}$. To estimate the forecasts in the original scale, inversion such as exponentiation of the $\log$ mortality forecasts is required. Once the forecast of age and gender specific mortality rates are obtained, we use a standard actuarial life table approach to estimate the life expectancy at birth for each population (see for example [8] for the life table method in details).

\subsection{The Forecast Error Measures}

In order to evaluate the accuracy of the Lee-Carter model, the out-sample forecast errors of mortality rates and life expectancy at births are estimated and compared across countries. The 56-year observation rates from 1960 to 2015 are divided into two parts: an estimation part and an evaluation part. The estimation part consists of 30 years in-sample data from 1960 to 1989 and the evaluation part comprises the remaining 26-year out-sample data from 1990 to 2015. The 30-year data are fitted into the model to forecast the mortality rates for the next 26 years. The forecast values are then compared with observation rates to estimate errors. In this study, the out-sample forecast errors are estimated using two error measurements which are Mean Forecast Error (MFE) and Mean Absolute Percentage Forecast Error (MAPFE) described as the following.

$$
\begin{gathered}
M F E=\frac{\sum_{n=1}^{N} \sum_{j=1}^{p} F_{j, n}-y_{j, n}}{N \times P} \\
M A P F E=\frac{\sum_{n=1}^{N} \sum_{j=1}^{p}\left|\frac{F_{j, n-} y_{j, n}}{y_{j, n}}\right| \times 100}{N \times P}
\end{gathered}
$$

where $F_{j, n}$ is the mortality forecast value for people who are age $j$ at year $n$ and $y_{j, n}$ is its corresponding actual rate. The parameters $N$ and $P$ are the last value of age and year respectively. 


\section{RESULTS AND DISCUSSION}

\subsection{Mortality Index $\left(k_{t}\right)$ of Developing Countries}

We analyze the estimated $k_{t}$ patterns for each country separately for males and females. It is noteworthy that the $k_{t}$ variable is an important indicator to show how well the mortality of population improves over the years. It can be seen from Fig. 3 that the $k_{t}$ pattern is generally decreasing over the years for all population, which may be due to significant improvement in health care and medical services. Nonetheless, a linear pattern can only be seen for Malaysian males, Malaysian females, Indonesian males and Indonesian females. For Thailand males and females, the mortality index is fluctuated over time in which a sharp decrease can be seen after year 1980s for females. This pattern indicates the rate of mortality improvement in Thailand is not constant over time. Singaporean mortality indices show an exponentially decreasing pattern over times since 1960 for males and 1970 for females. Thenon-linear decreasing mortality index patterns in Thailand and Singapore seem to violate the Lee-Carter model assumption. Thus, the accuracy of forecast values for both countries may be jeopardized.
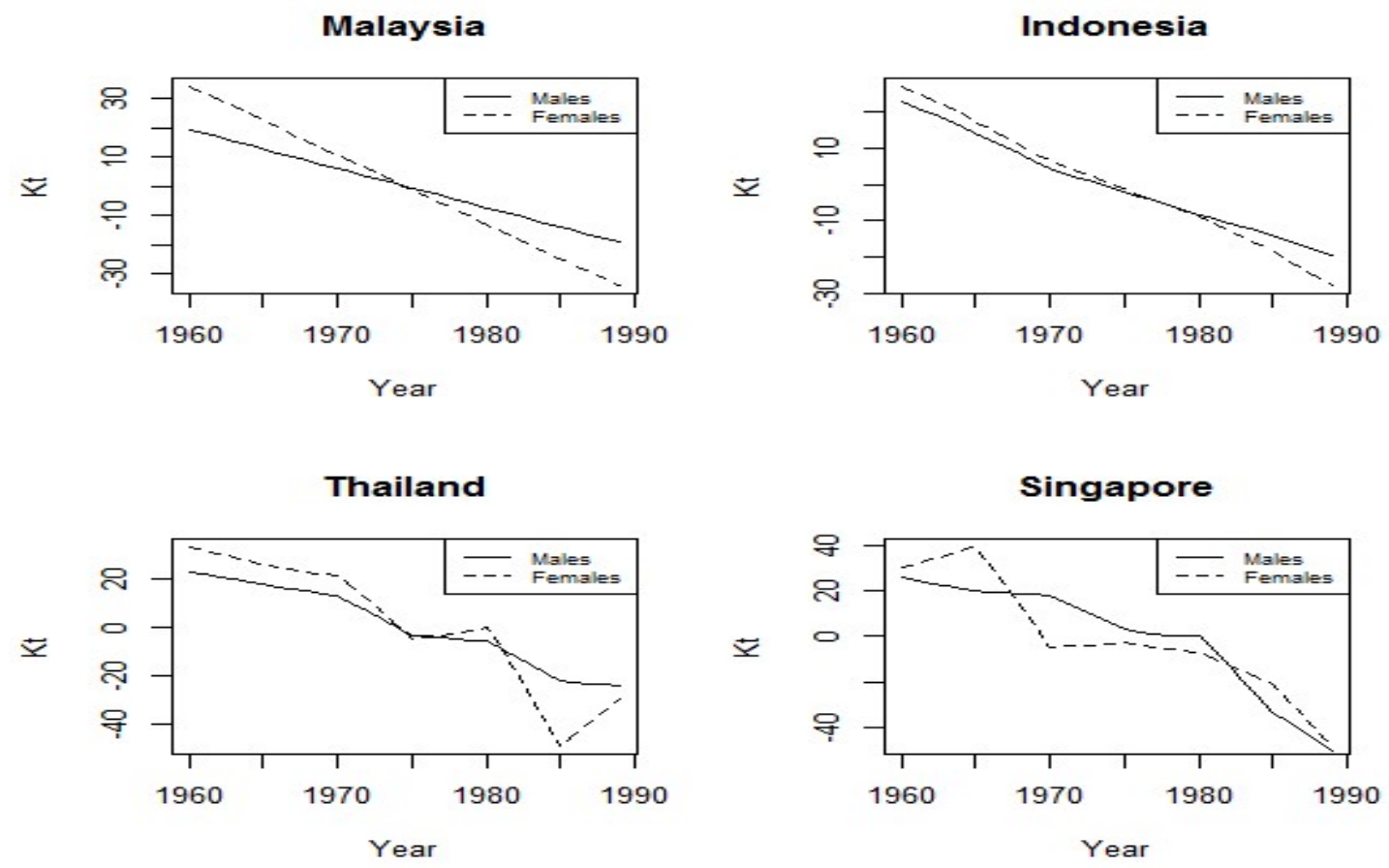

Fig.3. The estimated mortality index $k_{t}$ from the Lee-Carter model for males ( - ) and females (----) in Malaysia, Indonesia, Thailand and Singapore, from 1960 to 1989 


\subsection{Age and Gender Specific Mortality Forecast Errors}

We evaluate the performance of the Lee-Carter model to forecast age and gender specific mortality rates in four developing countries, Malaysia, Indonesia, Thailand and Singapore. The mortality rates were forecast over the out-sample period from 1990 to 2015 . The forecast values were then compared with the actual rates over the same period.
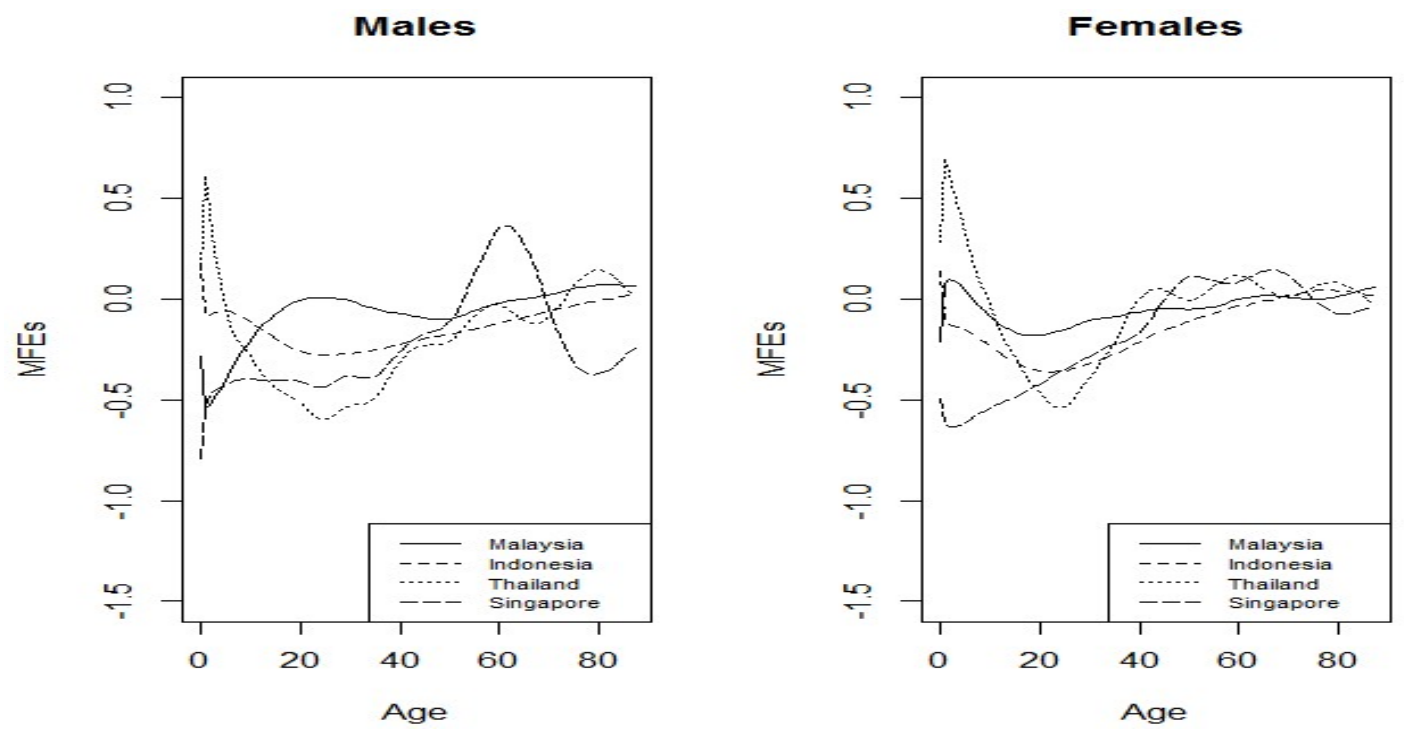

Fig.4. Mean Forecast Error (MFE) by age and countries for males (left panel) and females (right panel) in four developing countries

Fig. 4 presents mean forecast errors (MFEs) of mortality rates by age, gender and country. As can be seen from the right panel of Fig. 4, the Lee-Carter model works well for women between 40 and 85 years old due to the MFE values are fluctuated near zero value. The model overestimates the mortality rates of Thailand female infants and children from 0 to 10 years old and underestimates the mortality rates of Singapore females who are agedbetween 0 and 25 years old. The left panel of Fig. 4 shows the Lee-Carter model significantly overestimates the mortality rates of Singapore males who are aged between 45 and 60 years old and Thailand male children between 1 to 5 years old. Moreover, the model underestimates substantially the mortality rates of Singapore malesbetween 0 and 40 years old and Thailand males between 10 and 40 years old.

These results show that the Lee-Carter model may not be the best model to predict the mortality rates of Singapore and Thailand population. Highforecast errors fromthese population may be resulted from a non-linearity pattern of the estimated mortality index, $k_{t}$ 
asdescribed previously.According to $[9,5]$ to improve the forecast accuracy, an appropriate starting year of the fitting period must be optimally selected so that a reasonable linearly decreasing mortality index can be produced. Research from [10-12] proved that the accuracy of the Lee-Carter model was improved when using more recent starting year. Therefore, it is recommended for future works to estimate the best starting year of the Lee-Carter model fitting period for Thailand and Singapore.

Table 1.Mean Forecast Errors (MFEs) and Mean Absolute Percentage Forecast Error (MAPFEs) of log mortality rates for males and females by country

\begin{tabular}{ccccc}
\hline & \multicolumn{3}{c}{ Males } & \multicolumn{2}{c}{ Females } \\
Country & MFE & MAPFE(\%) & MFE & MAPFE(\%) \\
\hline Malaysia & -0.065 & 1.923 & -0.048 & 1.379 \\
Indonesia & -0.139 & 2.954 & -0.141 & 2.793 \\
Thailand & -0.194 & 3.052 & -0.047 & 3.144 \\
Singapore & -0.228 & 6.414 & -0.172 & 3.628 \\
\hline
\end{tabular}

Table 1summarizes the corresponding mean forecast errors (MFEs) and Mean Absolute Percentage Forecast Errors (MAPFEs) in which the mean is taken over age groups and years. The Lee and Carter model underestimates male and female mortality rates in all four developing countries in which the highest MFEs is recorded for Singapore males. In terms of absolute percentages, we can see from Table 1 that the MAPFE values are relatively small which are below 5\% for all population except for Singapore males.

\subsection{Life Expectancy at Birth Forecast Errors}

Next, we evaluate the Lee-Carter model's accuracy using life expectancy at birth as the outcome measure. From the mortality rate forecasts, we estimated the life expectancy at births forecastsusing the standard actuarial life table approach. The life expectancy at birth forecasts are then compared to the actual values to estimate the out-sample forecast errors.

Fig5.shows that the Lee-Carter model overestimates the life expectancy at birth of males in all four countries. The Lee-Carter model produces more accurate life expectancy forecasts for Malaysian males compared to other countries, which has shown by a smaller gap between dashed (forecast) and full (actual) lines. The model however tends to significantly overestimate the rapid decrease in life expectancy for Thailand males which has occurred 
since year 1980. This result leads to the highest MFE and MAPFE values for Thailand males in the Table 2. From Table 2, Malaysia males have the least forecast errors compared to males from other countries, which are 0.415 for MFE and only $0.598 \%$ for MAPFE.
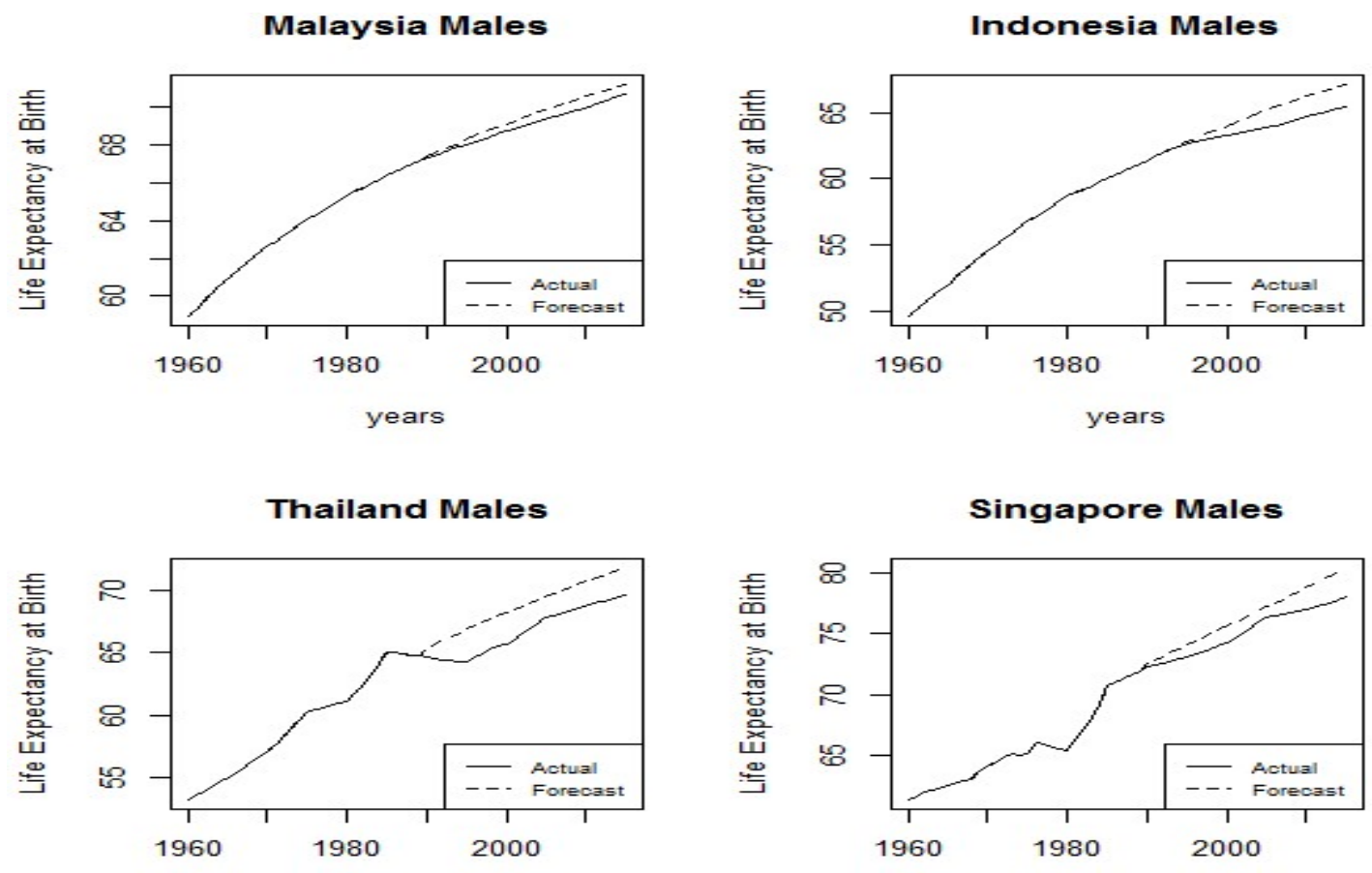

Fig.5.Life expectancy at birth actual values (-) and forecasts (-----) for males in Malaysia, Indonesia, Thailand and Singapore 


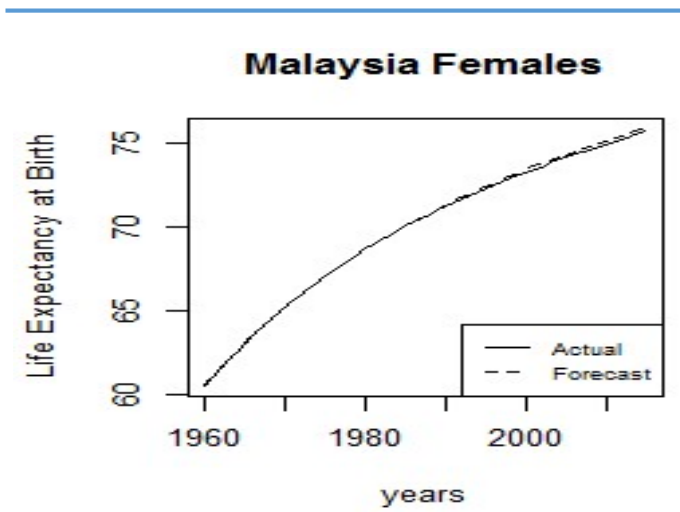

Thailand Females

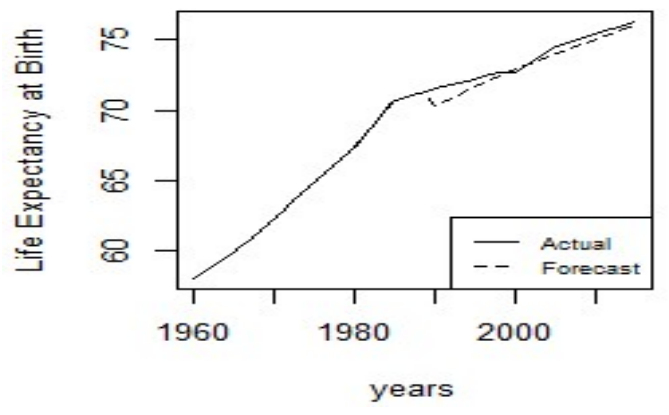

Indonesia Females

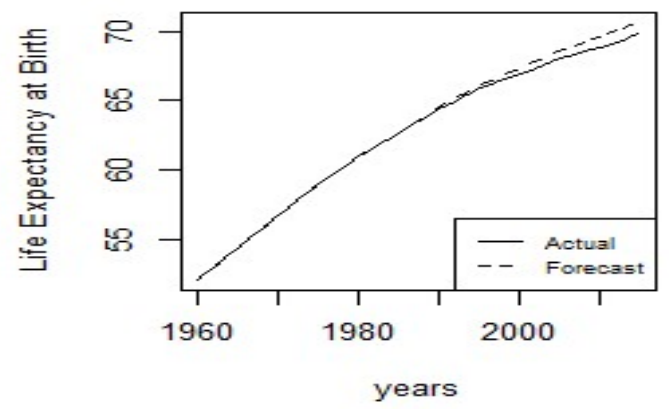

Singapore Females

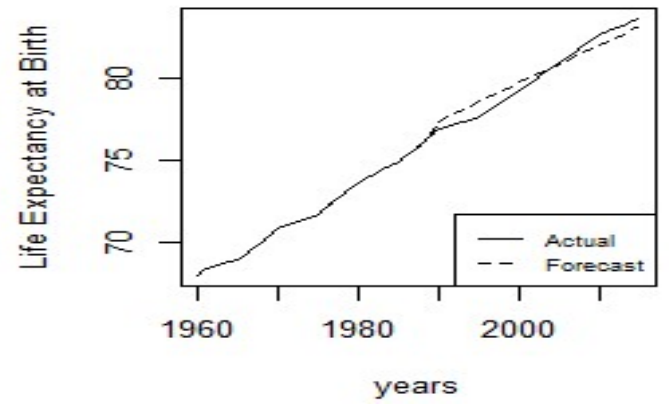

Fig.6. Life expectancy at birth actual values (-) and forecasts (----) for females in Malaysia, Indonesia, Thailand and Singapore

We separately forecast the life expectancy at births for females. Interestingly, Fig. 6 shows that the Lee-Carter model has the ability to produce more accurate life expectancy forecasts for females compared to males. It can be seen from the Fig. 6 that the life expectancy forecast values are closed to and sometimes touched the actual curves. The corresponding MFE and MAPFE results which presented in Table 2shows that the Lee-Carter model accurately forecasts life expectancy at birth of females in all four developing countries in which the MFE and MAPFE values are consistently less than that of males. 
Table 2.Mean Forecast Errors (MFEs) and Mean Absolute Percentage Forecast Errors (MAPFEs) of life expectancy at birth for males and females by country

\begin{tabular}{ccccc}
\hline & \multicolumn{2}{c}{ Males } & \multicolumn{2}{c}{ Females } \\
Country & MFE & MAPFE(\%) & MFE & MAPFE(\%) \\
\hline Malaysia & 0.415 & 0.598 & 0.156 & 0.211 \\
Indonesia & 0.934 & 1.455 & 0.496 & 0.728 \\
Thailand & 2.034 & 5.183 & -0.434 & 0.614 \\
Singapore & 1.282 & 1.693 & 0.125 & 0.656 \\
\hline
\end{tabular}

\section{CONCLUSION}

In this research, we forecast age and gender specific mortality rates and life expectancy at births of four developing countries using the well-known Lee-Carter model and report themodel's out-sample forecast errors. The examination of the mortality index patterns between countries shows that Thailand males and females and Singapore males and females displayed nonlinearity mortality indices over time. It is opened for future research to determine the best starting year of fitting period for these population so that a more linear mortality index can be produced. The out-sample forecast errors of mortality rates showed that the Lee-Carter model is not the best mortality model for Singapore males due to its higher errors compared to other populations. Moreover, the Lee-Carter model significantly overestimated the life expectancy of Thailand males. The forecast errors of life expectancy at births showed that the Lee-Carter model consistently performs better forecasts for females compared to males. For future works, it would be interesting if the extended versions of the Lee-Carter model can be explored using developing countries' data and compare the forecast performance between different models.

\section{ACKNOWLEDGEMENTS}

We would like to thank the Faculty of Computer and Mathematical Science, UiTM Shah Alam, for giving us the permission to present this paper in a conference and providing funds for registration fees. 


\section{REFERENCES}

[1] Booth H, Tickle L. Mortality modelling and forecasting: A review of methods. Annals of Actuarial Science, 2008, 3(1-2):3-43

[2] Pollard J H. Projection of age-specific mortality rates. Population Bulletin of the United Nations, 1998, 21-22:55-69

[3] Lee R D, Carter L R. Modelling and forecasting U.S. mortality. Journal of the American Statistical Association, 1992, 87(419):659-671

[4] Li N, Lee R D.Coherent mortality forecasts for a group of population: An extension of the Lee-Carter method. Demography, 2005, 42(3):575-594

[5] Booth H, Maindonald J, Smith L.Applying Lee-Carter under condition of variable mortality decline. Population Studies: A Journal of Demography,2002, 56(3):325-336

[6] United NationsDepartment of Economic and Social Affairs, Population Division (UN DESA).World population prospects: The 2015 revision.Geneva: UN DESA, 2015

[7] World Health Organization (WHO).Global status report on road safety: Time for action. Geneva: WHO, 2009

[8] Siegel J. S., Swanson D. A. The methods and materials of demography. London: Elsevier Academic Press, 2004

[9] Li J.A Poisson common factor model for projecting mortality and life expectancy jointly for females and males. Population Studies, 2013, 67(1):111-126

[10] Lee R, Miller T.Evaluating the performance of Lee-Carter method for forecasting mortality. Demography,2001, 38(4):537-549

[11] Tuljapurkar S, Li N, Boe C.A universal pattern of mortality decline in the G7 countries. Nature, 2000, 405(6788):789-792

[12] Hyndman R J, Booth H, Yasmeen F.Coherent mortality forecasting: The product-ratio method with functional time series models. Demography, 2013, 50(1):261-283

\section{How to cite this article:}

Shair S, Akmal F and Nordin N A. Multi-developing-country Comparison of The Lee-Carter Model for Mortality and Life Expectancy Projections. J. Fundam. Appl. Sci., 2017, 9(6S), 371-383. 